\title{
Descompresión endoscópica del túnel cubital, técnica y experiencia
}

\author{
V. Carratalá, F. Lucas, E. Sánchez Alepuz, R. Calero \\ Servicio de Traumatología. Unión de Mutuas. Valencia. España.
}

\begin{abstract}
Resumen: Objetivo: Presentar la técnica de liberación endoscópica del nervio cubital en el codo así como los resultados obtenidos en nuestra experiencia.

Material y métodos: Se recogieron prospectivamente datos de 16 pacientes a los que se realizó una descompresión endoscópica del nervio cubital en el codo en un período de tres años. Se empleó la clasificación de McGowan modificada y la electromiografía para el estadiaje preoperatorio. Los resultados postoperatorios fueron evaluados mediante la clasificación de Wilson \& Krout y la escala de valoración de la Clínica Mayo. En la clasificación preoperatoria un paciente $(6,25 \%)$ correspondía al grado I, $9(56,2 \%)$ al grado 2 A, 4 (25\%) al grado 2 B y 2 (I2,5\%) al grado 3.

Resultados: 6 pacientes (37,5\%) presentaban resultados excelentes, 8 (50\%) buen resultado y 2 (I2,5\%) resultados regulares o aceptables según la clasificación de Wilson \& Krout. Según la escala de valoración de la Clínica Mayo, 12 pacientes (75\%) presentaban resultados excelentes, 3 (I8,75\%) buenos resultados y I $(6,25 \%)$ resultado regular. El tiempo medio desde la cirugía hasta la reincorporación laboral fue de 36 días (rango de 17 a 52).

Conclusión: La descompresión endoscópica del nervio cubital es una técnica poco invasiva, segura, fiable y con buenos resultados.
\end{abstract}

Palabras claves: Nervio cubital, neuropatía, túnel cubital, descompresión endoscópica.

\begin{abstract}
Objetive:The aim of this study is to present the endoscopic cubital tunnel release technique and the results of this technique in our experience.

Methods: Prospective data of 16 patients were collected ( 9 males, 7 females, mean age 48 years) who underwent endoscopic cubital tunnel release during a period of 3 years. Modified McGowan's classification and electromyography was used for preoperative scoring. The postoperative results were scored using the Wilson \& Krout classification and the Mayo Elbow Performance Score System. Preoperatively I patient (6,25\%) had grade I, 9 patients (56,2\%) had grade 2A, 4 patients (25\%) had grade $2 \mathrm{~B}$ and 2 patients (12,5\%) had grade 3 .

Results:There were excellent results in 6 patients (37,5\%), good results in $8(50 \%)$, and fair results in $2(12,5 \%)$ according to Wilson \& Krout classification. According to Mayo Elbow Performance Score there were excellent results in 12 patients (75\%), good results in $3(18,75 \%)$ and fair results in I $(6,25 \%)$. The mean time between surgery and they retum to work was 36 days (17-52).

Conclusion:The endoscopic cubital tunnel release is a minimal invasive technique, safe, reliable and with good results.
\end{abstract}

Key words: ulnarnerve, endoscopic release, neuropathy, cubital tunnel syndrome.

La compresión del nervio cubital a nivel del codo es la neuropatía compresiva más frecuente en el miembro superior por detrás del síndrome del túnel carpiano ${ }^{1,2}$. La posición superficial del nervio en el túnel cubital y el aumento de tensión y tracción que experimenta con la flexión del codo, lo hacen muy susceptible a esta neuropatía compresiva.

En la mayoría de los pacientes el origen es idiopático, aunque hay que descartar lesiones como osteofitos, cúbito valgo postraumático, tumoraciones de partes blandas (gangliones...), contracturas postraumáticas y subluxación del nervio cubital en el epicóndilo medial ${ }^{3}$, pudiendo estar implicadas diferentes estructuras. Las localizaciones más frecuentes de compresión son el túnel cubital (más frecuente), formado por el epicóndilo medial, el cúbito proximal, el límite tendinoso del flexor carpi ulnaris y la fascia entre éste y el ligamento arcuato de Osborne. A nivel proximal, la zona de mayor compresión es la arcada de Struthers. Las localizaciones de las posibles compresiones se extienden desde unos $8 \mathrm{~cm}$ proximales a unos $5 \mathrm{~cm}$ distales al epicóndilo medial².

Las parestesias en el borde cubital de la mano y antebrazo son el hallazgo más común. Los pacientes tam-
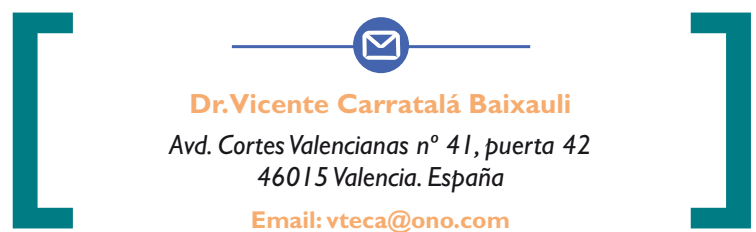


\begin{tabular}{|c|c|}
\hline $\begin{array}{l}\text { TABLA } \\
\text { TADIFICAC } \\
\text { EL CODO }\end{array}$ & $\begin{array}{l}\text { LASIFICACIÓN DE MCGOWAN MODIFICADA }{ }^{7} \text { DE ES- } \\
\text { N PREOPERATORIA DE LA NEUROPATÍA CUBITAL EN }\end{array}$ \\
\hline Grado & Descripción \\
\hline 1 & $\begin{array}{l}\text { Pacientes con síntomas subjetivos sensitivos pero sin ha- } \\
\text { llazgos objetivos }\end{array}$ \\
\hline $2 \mathrm{~A}$ & $\begin{array}{l}\text { Pacientes con buena fuerza intrínsecos }(4 / 5) \text {, sin atrofia in- } \\
\text { trínsecos }\end{array}$ \\
\hline 2B & Pacientes con moderada fuerza intrínsecos (3/5), con atrofia \\
\hline 3 & $\begin{array}{l}\text { Pacientes con marcada atrofia intrínsecos y alteraciones } \\
\text { sensitivas }\end{array}$ \\
\hline
\end{tabular}

bién refieren con frecuencia dolor en la cara medial del codo que irradia hacia el antebrazo, así como debilidad en la musculatura intrínseca y menor fuerza de prensa. En los casos severos y de larga evolución puede aparecer atrofia de la musculatura intrínseca, sobre todo en el primer músculo interóseo dorsal ${ }^{3}$.

El fallo del tratamiento conservador es indicación para realizar la liberación del nervio cubital a nivel del codo. Diferentes metaanálisis han concluido que la descompresión cubital simple tiene resultados comparables a la transposición anterior pero con menos complicaciones que esta última ${ }^{4,5}$. La descompresión endoscópica es tan efectiva como la cirugía abierta, y además cuenta con las ventajas de ser menos invasiva, preserva mejor la vascularización del nervio y la recuperación es más rápidal, 6 .

El propósito de este trabajo es mostrar los resultados que hemos obtenido realizando la descompresión endoscópica del nervio cubital a 16 pacientes en nuestro centro. Hemos realizado un seguimiento de los mismos desde el momento de la intervención quirúrgica hasta la reincorporación a su actividad laboral y/o deportiva. Hemos empleado la clasificación de McGowan modificada ${ }^{7}$ (T) Tabla I y la electromiografía para determinar el estadio inicial de cada paciente, y hemos cuantificado los resultados con la clasificación de Wilson \& Krout $^{8}$ @ Tabla II y la escala de valoración de codo de la Clínica Mayo (Mayo Elbow Performance Score). Se realizaron nuevas revisiones a los 6 meses y al año tras la cirugía para estudiar posibles recidivas.

\section{MATERIALY MÉTODO}

Se han recogido prospectivamente los datos de 16 pacientes a los que se realizó una descompresión en-

\begin{tabular}{|c|c|}
\hline $\begin{array}{l}\text { TABLA II - } \\
\text { ESTADIFICA } \\
\text { NEUROPATÍ }\end{array}$ & $\begin{array}{l}\text { CRITERIOS DE WILSON \& KROUT }{ }^{8} \text { PARA LA } \\
\text { IÓN POSTOPERATORIA EN PACIENTES CON } \\
\text { CUBITAL }\end{array}$ \\
\hline Grado & Descripción \\
\hline Excelente & $\begin{array}{l}\text { No dolor en zona de la incisión, mínimas alteraciones } \\
\text { sensitivas y motoras }\end{array}$ \\
\hline Bueno & $\begin{array}{l}\text { Pérdida de los síntomas de la neuropatía pero alteracio- } \\
\text { nes sensitivas ocasionales }\end{array}$ \\
\hline Regular & $\begin{array}{l}\text { Mejoría, pero persisten alteraciones sensitivas o motoras, } \\
\text { que son menores que antes de la intervención }\end{array}$ \\
\hline Malo & No mejoría o empeoramiento \\
\hline
\end{tabular}

cópica del nervio cubital en el codo en un período de tres años@Tabla III.

Todos los pacientes fueron intervenidos por el mismo cirujano y utilizando la misma técnica. De los 16 pacientes intervenidos 9 de ellos eran varones $(56,2 \%)$ y 7 correspondían a mujeres (43,8\%). La edad media se situó en 48 años con un rango de 24 a 64 años, siendo la mediana de 49 años. El codo derecho se vio afectado en 12 pacientes (75\%) frente a las 4 lesiones (25\%) en el lado izquierdo. El tiempo medio desde el inicio de los síntomas hasta el tratamiento quirúrgico fue de 9 meses y 12 días con un rango desde los 6 hasta los 18 meses. Los pacientes eran trabajadores manuales y/o deportistas.

Tras la anamnesis y exploración física sugestiva de neuropatía cubital se confirmó el diagnóstico mediante estudio electromiográfico, que reveló atrapamientos severos en 6 de los pacientes (37,5\%), un atrapamiento moderado/severo en otros 6 pacientes $(37,5 \%)$ y atrapamientos moderados en 4 pacientes (25\%). La mayor parte de los pacientes con afectación severa correspondían a pacientes del sexo femenino con una relación mujer-varón de 5/I aunque no existieron diferencias estadísticamente significativas entre ambos sexos.

Los pacientes se clasificaron preoperatoriamente en 4 grados siguiendo la clasificación modificada de McGowan ${ }^{7}$, en la que un paciente $(6,25 \%)$ correspondía al grado I, 9 pacientes (56,2\%) al grado 2A, 4 pacientes $(25 \%)$ al grado $2 \mathrm{~B}$ y 2 pacientes $(12,5 \%)$ al grado 3.

Se realizaron radiografías para descartar patología ósea concomitante. 
TAala III- Pacientes intervenidos con datos Registrados.

\begin{tabular}{|c|c|c|c|c|c|c|c|c|c|}
\hline Pacientes & SEXO & EDAD & LAT & $\begin{array}{l}\text { INICIO } \\
\text { (meses) }\end{array}$ & EMG & $\begin{array}{c}\text { Mc } \\
\text { Gowan }\end{array}$ & $\begin{array}{c}\text { DIAS HASTA } \\
\text { ALTA }\end{array}$ & $\begin{array}{l}\text { Wilson- } \\
\text { Krout }\end{array}$ & MEPS \\
\hline 1 & V & 56 & DER & 6 & MOD & $2 \mathrm{~A}$ & 24 & EX & 100 \\
\hline 2 & $\mathrm{M}$ & 24 & IZQ & 8 & M/SEV & $2 \mathrm{~B}$ & 36 & B & 90 \\
\hline 3 & V & 45 & DER & 9 & MOD & $2 \mathrm{~A}$ & 28 & EX & 100 \\
\hline 4 & V & 61 & DER & 10 & M/SEV & $2 A$ & 17 & B & 100 \\
\hline 5 & V & 61 & IZQ & 10 & M/SEV & $2 \mathrm{~A}$ & 26 & B & 85 \\
\hline 6 & $M$ & 38 & $\mathrm{IZQ}$ & 12 & SEV & $2 \mathrm{~B}$ & 52 & B & 80 \\
\hline 7 & V & 43 & DER & 9 & SEV & $2 \mathrm{~A}$ & 37 & EX & 100 \\
\hline 8 & V & 49 & DER & 8 & MOD & $2 \mathrm{~A}$ & 46 & $\mathrm{~B}$ & 90 \\
\hline 9 & $M$ & 56 & DER & 7 & SEV & $2 \mathrm{~A}$ & 42 & EX & 100 \\
\hline 10 & M & 33 & DER & 6 & MOD & 1 & 31 & EX & 100 \\
\hline 11 & V & 64 & DER & 12 & M/SEV & $2 \mathrm{~B}$ & 51 & $\mathrm{~B}$ & 90 \\
\hline 12 & $M$ & 49 & DER & 18 & SEV & 3 & 47 & REG & 70 \\
\hline 13 & V & 54 & DER & 8 & M/SEV & $2 \mathrm{~A}$ & 37 & EX & 100 \\
\hline 14 & V & 56 & DER & 9 & M/SEV & $2 \mathrm{~B}$ & 35 & B & 100 \\
\hline 15 & M & 44 & IZQ & 12 & SEV & 3 & 37 & REG & 85 \\
\hline 16 & M & 36 & DER & 7 & SEV & $2 \mathrm{~A}$ & 35 & B & 100 \\
\hline MEDIA & & & & 9,44 & & & 36,31 & & \\
\hline
\end{tabular}

B (Buenos), Der (Derecha), emg (Electromiogafa), EX (Excelentes), IZQ (Izquierda), Lat (Lateraldad), M (Mujer), Meps (Mayo Elbow Performance Score), mod (Moderado), M/SEV (Moderedo/Severo), Reg (Regular), SeV (Severo), V (Varón).

Se indicó la liberación endoscópica en aquellos casos en los que los síntomas no habían mejorado tras 3 meses de tratamiento conservador. El período de duración de los síntomas hasta la cirugía fue de 9,43 meses. Se excluyeron del estudio aquellos pacientes con antecedentes y secuelas de fractura en el codo afecto, pacientes con rigidez articular que precisaron artrolisis de codo asociada a la liberación cubital y aquellos con radiculopatía cervical asociada. Se empleó el bloqueo axilar como anestesia en todos los pacientes e isquemia del brazo intervenido.

La descompresión endoscópica del nervio cubital se realizó siguiendo la técnica descrita por Cobb6. Mediante una pequeña incisión de $1,5 \mathrm{~cm}$ localizada a $1 \mathrm{~cm}$ proximal al canal retroepitroclear se localizó el nervio cubital. Empleando los dilatadores y vainas del sistema Endorelease Endoscopic Cubital Tunnel Release System ${ }^{\circ}$ (Integra LifeSciences, Plainsboro, NJ) (2) Figura I, y con la óptica a través de la vaina podemos visualizar el nervio cubital tanto en su recorrido proximal al codo como en el distal (1) Figuras 2, 3 y 4. Con la ayuda de una cuchilla se realizó la descompresión del nervio en ambas direcciones. Debe avanzarse con la cuchilla al mismo tiempo que con la óptica, de esta forma el nervio queda siempre protegido ya que la óptica se interpone entre la cuchilla y el nervio cubital (2) Figura 5. Previamente se ha comprobado mediante visión endoscópica que en el recorrido que vamos a liberar no hay ningún vaso ni rama nerviosa.

Una vez realizada la descompresión se comprobó con la óptica que el nervio estaba liberado y no presentaba otros puntos de compresión en su recorrido (1) Figura 6.

Tras retirar la isquemia y realizar hemostasia se suturó la incisión de la piel y se inyectó a través de un pequeño tubo de drenaje, que posteriormente se retiró, anestésico local y vasoconstrictor (Svedocain 0,25\%: Bupivacaína hidrocloruro+epinefrina) para reducir el sangrado. Se aplicó un vendaje y un cabestrillo.

Los pacientes iniciaron la movilización del codo al día siguiente de la cirugía. El vendaje se retiró a los 7 días iniciando entonces el tratamiento de rehabilitación.

Los resultados postquirúrgicos se evaluaron mediante la escala de Wilson \& Krout modificada ${ }^{8}$ y con la escala MEPS (Mayo Elbow Performance Score).

Los datos se analizaron con el programa IBM SPSS Statistics for Windows, versión PASW stadistics 17 por un estadístico ajeno a los autores del artículo. 


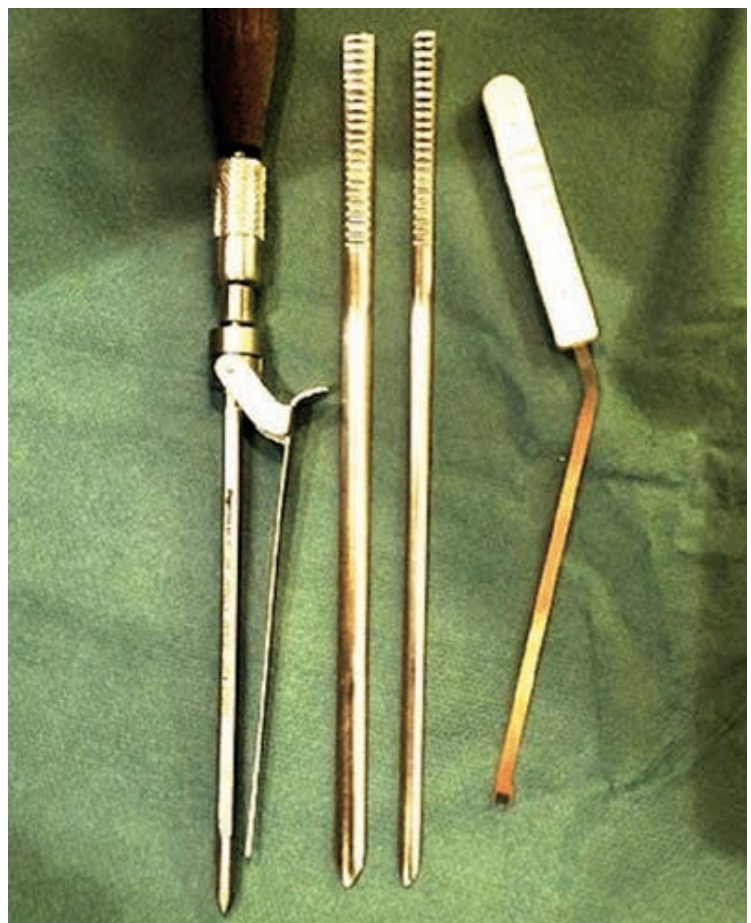

Figura I. Cánula y material del sistema Endorelease ${ }^{\circledR}$.

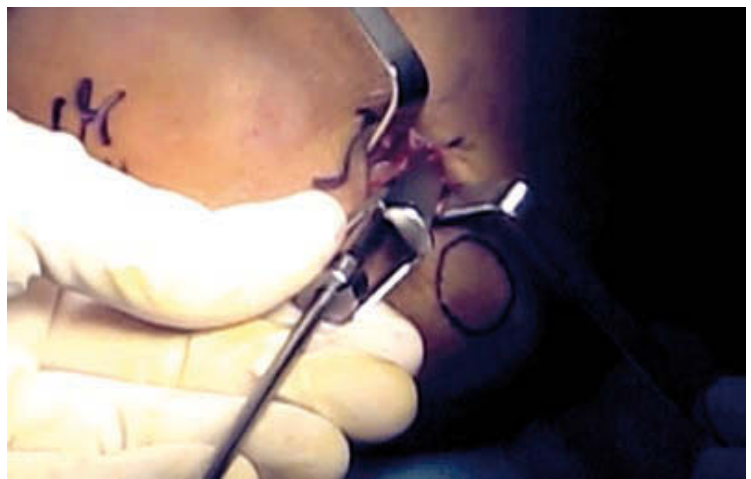

Figura 2. Introducción de la cánula y óptica tras la localización del nervio.

Se consideró estadísticamente significativo un valor de $p<0,05$.

\section{RESULTADOS}

Los resultados postquirúrgicos, (a) Tabla IV, tras la liberación endoscópica del nervio cubital, mostraron 6 pacientes $(37,5 \%)$ con resultados excelentes, 8 pacientes (50\%) con un buen resultado y 2 pacientes ( $12,5 \%)$ con resultados regulares o aceptables. Los 2 resultados menos favorables correspondían a los pacientes con mayores tiempos de sintomatología clí-

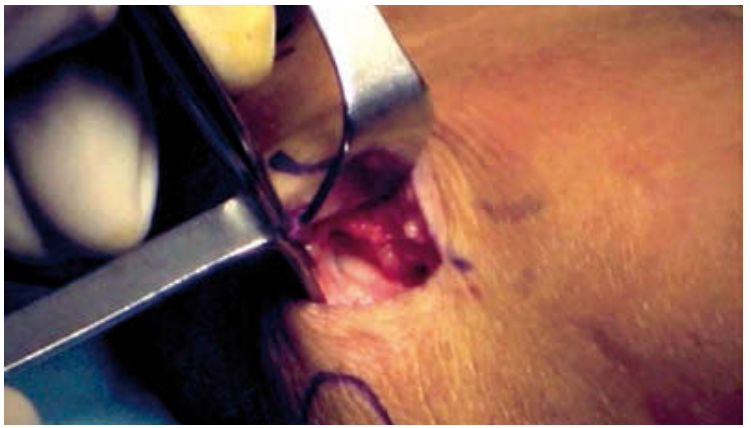

Figura. 3. Visión del nervio en su trayecto proximal.

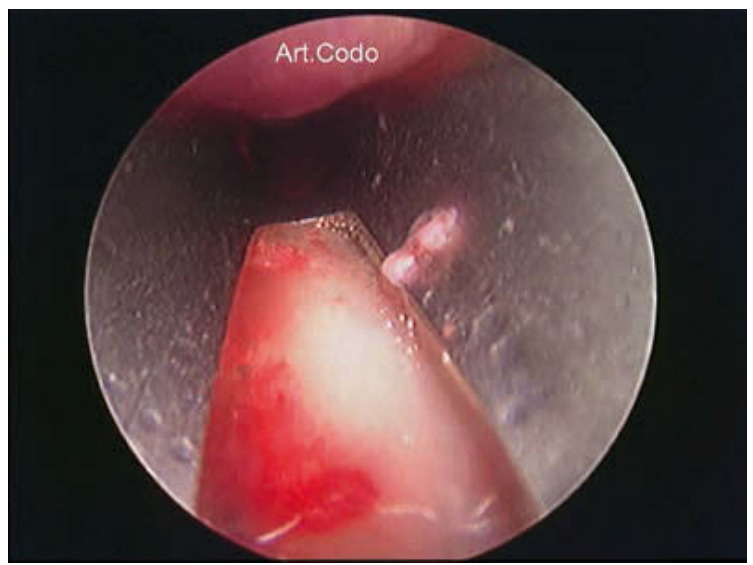

Figura 4. Visión endoscópica del nervio cubital.

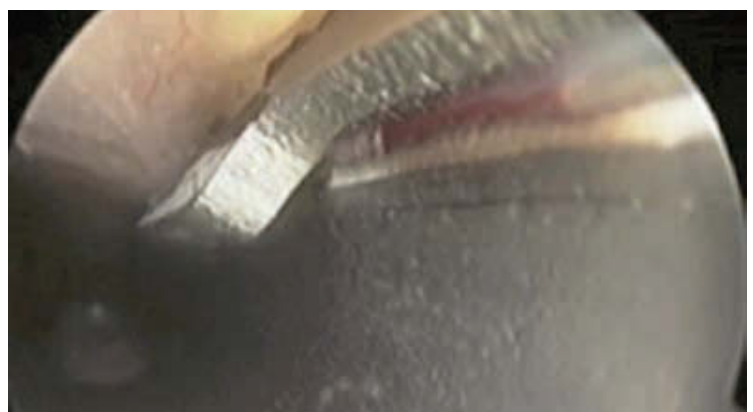

Figura 5. Visión endoscópica de la liberación nerviosa. En la parte superior se observa la cuchilla empleada.

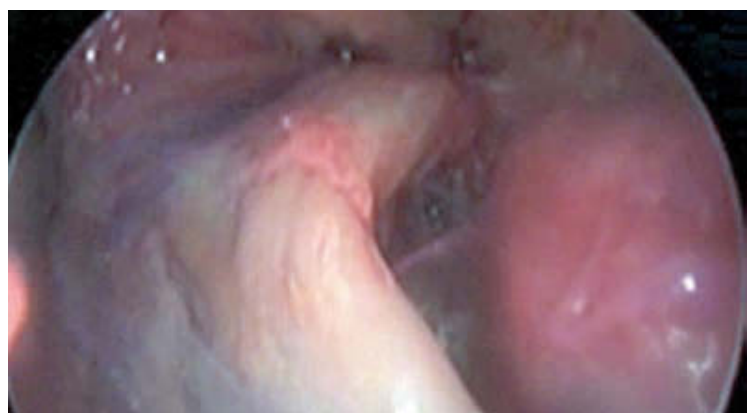

Figura 6. Visión endoscópica del nervio liberado. 


\begin{tabular}{|c|c|c|c|c|c|}
\hline \multicolumn{6}{|c|}{$\begin{array}{l}\text { TABLA IV - RESULTADOS POSTOPERATORIOS SEGÚN LA CLASIFICACIÓN DE WILSON \& KROUT COMPARADOS CON LOS RESULTADOS } \\
\text { PREOPERATORIOS SEGÚN LA CLASIFICACIÓN DE McGOWAN Y LA ELECTROMIOGRAFía (EMG). }\end{array}$} \\
\hline \multirow[b]{2}{*}{$\begin{array}{l}\text { CLASIFICACIÓN PREOPERATORIA } \\
\text { DE MCGOWAN }\end{array}$} & \multicolumn{5}{|c|}{ CLASIFICACIÓN DE WILSON \& KROUT MODIFICADA } \\
\hline & Excelente & Bueno & Regular & Malo & TOTAL \\
\hline Grado I & $1(6,25 \%)$ & & & & $1(6,25 \%)$ \\
\hline Grado IIA & $5(31,25 \%)$ & $4(25 \%)$ & & & $9(56,2 \%)$ \\
\hline Grado IIB & & $4(25 \%)$ & & & $4(25 \%)$ \\
\hline Grado III & & & $2(12,5 \%)$ & & $2(12,5 \%)$ \\
\hline TOTAL & $6(37,5 \%)$ & $8(50 \%)$ & $2(12,5 \%)$ & & 16 \\
\hline \multicolumn{6}{|l|}{$\begin{array}{l}\text { Electromiograma (EMG) } \\
\text { PREOPERATORIO }\end{array}$} \\
\hline Severo & $2(12,5 \%)$ & $2(12,5 \%)$ & $2(12,5 \%)$ & & $6(37,5 \%)$ \\
\hline Moderado/Severo & $1(6,25 \%)$ & $5(31,25 \%)$ & & & $6(37,5 \%)$ \\
\hline Moderado & $3(18,75 \%)$ & $1(6,25 \%)$ & & & $4(25 \%)$ \\
\hline TOTAL & $6(37,5 \%)$ & $8(50 \%)$ & $2(12,5 \%)$ & & 16 \\
\hline
\end{tabular}

nica con 12 y 18 meses respectivamente que además presentaban un grado de atrapamiento severo en la electromiografía y un grado 3 en la clasificación de McGowan.

De acuerdo con la escala MEPS (2) Tabla V, estos resultados se correspondían con 12 pacientes (75\%) con resultados excelentes, 3 pacientes ( $8,75 \%$ ) con buenos resultados y I paciente $(6,25 \%)$ con un resultado regular que correspondía al paciente con la mayor duración de la sintomatología (18 meses).

El tiempo medio desde la intervención quirúrgica hasta la reincorporación laboral fue de 36 días con un rango de 17 a 52 días. El procedimiento dio como resultado un alivio postoperatorio temprano de los síntomas y la buena satisfacción del paciente. Todos los pacientes mejoraron sintomáticamente una semana después de la cirugía y se mostraron satisfechos con el procedimiento.

Cuando analizamos estadísticamente la relación entre clasificación de Wilson \& Krout y la electromiografía preoperatoria (1) Tabla IV mediante un test exacto de Fisher, los resultados no fueron estadísticamente significativos $(p=0,178)$ si bien sí que existía cierta dependencia entre ambas variables cuando la electromiografía presentaba una afectación medio-severa con un buen resultado y cuando la electromiografía mostraba un atrapamiento severo con resultados aceptables. Sin embargo, al comparar la clasificación de Wilson y Krout con la clasificación de McGowan sí que aparecieron relaciones estadísticamente significativas $(p=0,002)$ entre ambas variables. Existía además, al igual que en la comparación con la electromiografía, una tendencia positiva (más casos observados que esperados) entre el grado III de la clasificación de McGowan con resultados aceptables en la clasificación de Wilson y Krout y también entre el grado 2B de McGowan y un buen resultado final. Hemos aplicado el test exacto de Fisher para calcular el grado de significación $\mathrm{P}$, porque no se cumplen las condiciones de aplicación del chi-cuadrado de Pearson al analizar las dos variables categóricas, ya que alguna de las frecuencias esperadas es inferior a 5 y la muestra es demasiado pequeña.

No se registró ninguna complicación tras la cirugía y todos los pacientes regresaron a sus actividades previas, incluyendo el trabajo y la práctica deportiva.

En las revisiones posteriores a los 6 meses y al año no se detectó ninguna recidiva o agravamiento de la sintomatología y no hubo que reintervenir a ningún paciente. 


\begin{tabular}{|c|c|c|c|c|c|}
\hline \multirow[b]{2}{*}{$\begin{array}{l}\text { CLASIFICACIÓN PREOPERATORIA } \\
\text { DE MCGOWAN }\end{array}$} & \multicolumn{5}{|c|}{ CLASIFICACIÓN MEPS (Mayo Elbow Performance Score) } \\
\hline & $\begin{array}{l}\text { Excelente } \\
90-100\end{array}$ & $\begin{array}{c}\text { Bueno } \\
75-89\end{array}$ & $\begin{array}{l}\text { Aceptable } \\
60-74\end{array}$ & $\begin{array}{c}\text { Malo } \\
<60\end{array}$ & TOTAL \\
\hline Grado I & 1 (6,25\%) & & & & $1(6,25 \%)$ \\
\hline Grado IIA & $8(50 \%)$ & $1(6,25 \%)$ & & & $9(56,2 \%)$ \\
\hline Grado IIB & $3(18,75 \%)$ & $1(6,25 \%)$ & & & $4(25 \%)$ \\
\hline Grado III & & $1(6,25 \%)$ & $1(6,25 \%)$ & & $2(12,5 \%)$ \\
\hline TOTAL & 12 (75\%) & 3 (18,75\%) & 1 (6,25\%) & & 16 \\
\hline \multicolumn{6}{|l|}{$\begin{array}{l}\text { Electromiograma (EMG) } \\
\text { PREOPERATORIO }\end{array}$} \\
\hline Severo & $3(18,75 \%)$ & $2(12,5 \%)$ & $1(6,25 \%)$ & & $6(37,5 \%)$ \\
\hline Moderado/Severo & $5(31,25 \%)$ & $1(6,25 \%)$ & & & $6(37,5 \%)$ \\
\hline Moderado & $4(25 \%)$ & & & & $4(25 \%)$ \\
\hline TOTAL & $12(75 \%)$ & $3(18,75 \%)$ & $1(6,25 \%)$ & & 16 \\
\hline
\end{tabular}

\section{DISCUSIÓN}

Existen numerosas técnicas de descompresión nerviosa para el tratamiento de la neuropatía compresiva del nervio cubital a nivel del codo, ya sean de cirugía abierta o endoscópica, y cada cirujano puede tener preferencia por una u otra pero todavía existe un gran debate sobre cuál de las técnicas descritas es la mejor para tratamiento quirúrgico de los pacientes con síndrome del túnel cubital. El síndrome del túnel cubital fue tratado inicialmente con la transposición subcutánea anterior en 1898. Autores posteriores describen la transposición intramuscular anterior en 1917, la descompresión simple en 1922, la transposición submuscular anterior en 1942 y la epicondilectomía medial. Las técnicas de liberación endoscópicas popularizadas por Bain \& Bajhau ${ }^{2}$, Tsai y cols , Cobb $^{10}$ y Mirza y cols" trataron de modificar las técnicas endoscópicas existentes para el túnel carpiano para ser utilizadas en el túnel cubital.Todas estas técnicas permiten la liberación del nervio cubital a nivel del codo visualizando directamente el trayecto nervioso con mínimas incisiones. La liberación endoscópica del nervio cubital pretende ofrecer al paciente varios beneficios en relación a la cirugía abierta, como reducir la morbilidad del área quirúrgica, minimizar el riesgo de lesión de las estructuras adyacentes, una recuperación más rápida y unos resultados estéticos más satisfactorios por el menor tamaño de la incisión ${ }^{12}$. Además de las ventajas esté- ticas de una incisión pequeña, las técnicas endoscópicas han demostrado ser seguras y eficaces 12, 13, 14, 15, 16 con resultados buenos y excelentes en alrededor de un $87 \%$ 8, $, 12,17$ de los pacientes. La eficacia y la seguridad de la liberación del túnel cubital endoscópica también han sido confirmadas por los estudios que se han realizado en cadáveres ${ }^{2}$. Ahcan \& Zorman ${ }^{18}$ presentaron una ligera modificación de la técnica de Hoffmann y Siemionow ${ }^{12}$ cuyos resultados en 47 pacientes con síndrome del túnel cubital unilateral fueron de buenos a excelentes en un $92 \%$ de sus pacientes con un seguimiento que incluía la mejora en los estudios electromiográficos. Estos resultados se corresponden completamente con los obtenidos mediante nuestra técnica que alcanzan un $94 \%$ de buenos y excelentes resultados y a su vez se correlacionan favorablemente con Hoffmann y la serie original de 76 nervios de Siemionow ${ }^{12}$ que publicaba una tasa global de buenos y excelentes resultados de un $94 \%$ a pesar de utilizar escaladas de clasificación distintas.

Para la realización de nuestro estudio, nosotros hemos utilizado la escala de Wilson \& Krout modificada por Heithoff y cols ${ }^{8}$ ya que además de los síntomas neurológicos típicos también analiza la clínica relacionada con la zona de incisión quirúrgica, tan importante para el resultado final según Ahcan \& Zorman'18. Al igual que en otros estudios, se obtuvieron los mejores resultados en el grupo de pacientes con síntomas 
preoperatorios leves y moderados ${ }^{8,9,12,17}$. Gervasio y cols $^{19}$, Biggs \& Curtis $^{20}$ y Bartels y cols ${ }^{21}$ han demostrado en estudios prospectivos que la liberación in situ del nervio cubital es al menos tan eficaz como la transposición submuscular o transposición subcutánea, lo que ha apoyado el uso y el desarrollo, en lo últimos años, de técnicas cada vez menos invasivas en el tratamiento del síndrome del túnel cubital que ofrecen al paciente un resultado satisfactorio. Dellon ${ }^{22}$ realizó un metaanálisis con la revisión de 50 estudios diferentes de más de 2.000 pacientes que utilizaban diversos tratamientos quirúrgicos. Llegó a la conclusión de que en el $90 \%$ de los pacientes con síntomas de compresión mínimos cualquier procedimiento quirúrgico es satisfactorio. La evidencia que apoya el papel de la liberación in situ es abundante demostrándose que se obtienen buenos y excelentes resultados entre el $75 \%$ y el $90 \%$ de los pacientes que se someten a una descompresión in situ.

Aunque Ward ${ }^{23}$ demostró que en pacientes con estudios electromiográficos normales o ligeramente positivos, la descompresión endoscópica del nervio cubital conseguía una mejoría en la clínica, las personas con debilidad motora o estudios electromiográficos moderados o severos no obtuvieron tan buenos resultados. Esto difiere en parte con los resultados obtenidos en nuestra técnica. Sí que es cierto que los resultados menos favorables se han obtenido en los pacientes con atrapamientos más severos pero también se han obtenido buenos y excelentes resultados en pacientes con estudios electromiográficos severos y moderados.

Ahcan y Zorman ${ }^{18}$ afirmaban que era necesaria la conversión de la cirugía endoscópica a una técnica abierta si durante el procedimiento se apreciaba que el nervio cubital era inestable después de la descompresión. En nuestro estudio ningún caso precisó la reconversión a cirugía abierta. Esta afirmación viene apoyada por la publicación de Macadam y cols. ${ }^{5}$ que no mostró resultados estadísticamente significativos entre la descompresión simple y la transposición nerviosa en otro metaanálisis.

Los resultados obtenidos por Tsai en su estudio fueron excelentes en el $42 \%$ de los codos, buenos en el $45 \%$, regulares en $11 \%$ y pobres en el $2 \%$. Se produjo recurrencia de los síntomas en 3 codos y no obtuvo complicaciones graves. Ahcan \& Zorman ${ }^{18}$ obtuvieron con el sistema de clasificación de Wilson \& Krout modificado por Heithoff y cols, excelentes resultados en 21 pacientes (58\%), buenos resultados en 12 pacientes (33\%) y 3 pacientes con resultados aceptables (8\%). Estos resultados son comparables con los obtenidos por nosotros ya que obtuvimos un $37,5 \%$ de pacientes con resultados excelentes, un $50 \%$ con buenos resultados y un $12,5 \%$ con resultados regulares o aceptables. Sin embargo, no obtuvimos ninguna recurrencia de la sintomatología y tampoco complicaciones graves. Entre las complicaciones del tratamiento quirúrgico convencional del síndrome del túnel cubital se incluían la lesión del nervio cutáneo antebraquial medial (NCAM) y el fracaso para descomprimir completamente el nervio. La reintervención para la descompresión fallida del nervio cubital en el codo, por lo general, revela zonas persistentes o adicionales de compresión, más frecuentemente a nivel del tabique intermuscular medial proximal y distalmente en la aponeurosis flexopronadora. Según se desprende del estudio anatómico detallado de la arcada de Struthers ${ }^{24}$, el sitio clínicamente relevante de atrapamiento se encuentra $9,6 \mathrm{~cm}$ proximal al epicóndilo medial, donde el nervio cubital atraviesa dicho tabique intermuscular. Actualmente ninguno de los pacientes incluidos en nuestro estudio ha precisado una nueva liberación ya que con la técnica descrita alcanzamos niveles muy proximales y distales que evitan una nueva compresión a nivel del tabique intermuscular medial proximal y de la aponeurosis distal. Las incisiones más grandes $(6-10 \mathrm{~cm})$ utilizadas en la liberación convencional pusieron en riesgo las ramas localizadas del nervio cutáneo antebraquial medial. En nuestro estudio tampoco hemos constatado lesiones de dicho nervio.

Los resultados obtenidos tras el análisis estadístico de los datos nos aportó información sobre la tendencia que existe entre los resultados postoperatorios obtenidos y el grado de afectación preoperatoria. Esto concuerda con que a mayor afectación preoperatoria se consiguen resultados postoperatorios menos favorables aunque sería necesario aumentar el tamaño de la muestra para obtener datos estadísticamente significativos.

La gran mayoría de estudios comparativos del uso de técnicas de liberación del túnel cubital con una mínima incisión está aumentando y todos los estudios existentes muestran un beneficio. Sin embargo, estos estudios están limitados por la falta de grupos de control, un tamaño de la muestra relativamente pequeño y medidas de resultados no estandarizadas. 


\section{CONCLUSIÓN}

Los resultados de nuestro estudio apoyan la recomendación de otros autores de realizar la liberación del túnel cubital mediante descompresión endoscópica ya que se trata de una técnica segura, fiable y con buenos resultados para el tratamiento del síndrome del túnel cubital en pacientes que no presenten las contraindicaciones previamente descritas. La técnica precisa del conocimiento previo y manejo de las técnicas artroscópicas.

\section{AGRADECIMIENTOS}

Agradecemos a Ana Zapater García su inestimable colaboración en el proceso y tratamiento de los datos estadísticos obtenidos.

\section{CONFLICTOS DE INTERESES}

Los autores declaran no tener conflictos de intereses.

\section{BIBLIOGRAFÍA}

I. Watts AC, Bain GI. Patient-rated outcomes of ulnar nerve decompression: a comparison of endoscopic and open in situ decompression. J Hand Surg Am. 2009;34(8): | 492-8.

2. Bain Gl, Bajhau A. Endoscopic release of the ulnar nerve at the elbow using the Agee device: a cadaveric study. Arthroscopy. 2005;21 (6):69 I-5.

3. Mitsionis GI, Manoudis GN, Paschos NK, Korompilias AV, Beris AE. Comparative study of surgical treatment of ulnar nerve compression at the elbow. J Shoulder Elbow Surg. 20 10; 19:5 1 3-9.

4. Zlowodzki M, Chan S, Bhandari M, Kalliainem L, Schubert W. Anterior transposition compared with simple decompression for treatment of cubital tunnel syndrome. A meta-analysis of randomized, controlled trials. J Bone Joint Surg Am. 2007;89( | 2):259|-8.

5. Macadam SA, Gandhi R, Bezuhly M, Lefaivre KA. Simple decompression versus anterior subcutaneous and submuscular transposition of the ulnar nerve for cubital tunnel syndrome: a meta-analysis. J Hand Surg Am. 2008;33(8): I 3 | 4 el-el 2.

6. CobbT, Lemke J, Tyler J,Sterbank P. Efficacy of endoscopic cubital tunnel release. Hand. 2008;3(2): I91.
7. Goldberg BJ, Light TR, Blair SJ. Ulnar neuropathy at the elbow: results of medial epicondylectomy. J Hand Surg Am. 1989; | 4: | 82-8.

8. Heithoff SJ, Millender LH, Nalebuff EA, Petruska AJ. Medial epicondylectomy for treatment of ulnar nerve compression at the elbow. J Hand Surg. 1990; I 5A:22-9.

9. Tsai TM, Chen IC, Majd ME, Lim BH. Cubital tunnel release with endoscopic assistance: results of a new technique. J Hand Surg Am. 1999;24(1):21-9.

10. Cobb TK. Endoscopic cubital tunnel release. J Hand Surg Am. 201 0;35 (10): 1 690-7.

I I. Mirza A, Reinhart MK, Bove J, Litwa J. Scope-assisted release of the cubital tunnel. J Hand Surg Am. 201 1;36(I):1475।.

12. Hoffmann R, Siemionow M. The endoscopic management of cubital tunnel syndrome. J Hand Surg Br. 2006:3। ( I):23-29.

13. Nakao Y, Takayama S, Toyama Y. Cubital tunnel release with lift-type endoscopic surgery. Hand Surg. 2001;6:199- 203.

14. Dellon AL, MacKinnon SE. Injury to the medial antebrachial cutaneous nerve during cubital tunnel surgery. J Hand Surg. 1985; I0B:33-6.

15. Taniguchi Y, Takami M, Takami T, Yoshida M. Simple decompression with small skin incisión for cubital tunnel syndrome. J Hand Surg. 2002;27B:559 -62.

16. Gabel GT, Amadio PC. Reoperation for failed decompression of the ulnar nerve in the region of the elbow. J Bone Joint Surg. 1990;72A:2 I3-9.

17. McGowan AJ. The results of transposition of the ulnar nerve for traumatic ulnar neuritis. J Bone Joint Surg Br. 1950;32-B(3):293-301.

18. Ahcan U, Zorman P. Endoscopic decompression of the ulnar nerve at the elbow. J Hand Surg Am. 2007;32(8): $1171-6$.

19. Gervasio O, Gambardella G, Zaccone C, Branca D. Simple decompression versus anterior submuscular transposition of the ulnar nerve in severe cubital tunnel syndrome: a prospective randomized study. Neurosurgery. 2005;56(I): I08 - 17.

20. Biggs M, Curtis JA. Randomized, prospective study comparing ulnar neurolysis in situ with submuscular transposition. Neurosurgery. 2006;58(2): $296-304$.

2I. Bartels RH, Verhagen WI, van der Wilt G], Meulstee J, van Rossum LG, Grotenhuis JA. Prospective randomized controlled study comparing simple decompression versus anterior subcutaneous transposition for idiopathic neuropathy of the 
ulnar nerve at the elbow: part I. Neurosurgery. 2005;56(3):522-30.

22. Dellon AL. Review of treatment results for ulnar nerve entrapment at the elbow. J Hand Surg. Am. 1989; | 4A:688-99.
23. Ward WA, Siffri PC. Endoscopically assisted ulnar neurolysis for cubital tunnel syndrome. Tech Hand Up ExtremSurg. 2009; I 3(3): I 55-9.

24. Von Schroeder HP, Scheker LR. Redefining the "Arcade of Struthers." J Hand Surg. 2003;28A: |0|8-2|. 